\title{
Vacinação antisarampo, parotidite e rubéola em crianças com suspeita de alergia ao ovo
}

Sónia Rosa, ${ }^{1}$ Filipa Ribeiro, ${ }^{2}$ Paula Leiria Pinto ${ }^{3}$

\section{RESUMO}

A Direção-Geral da Saúde (DGS) publicou uma orientação para a administração da vacina contra o sarampo, parotidite e rubéola (VASPR), tendo reservado a referenciação hospitalar para situações de anafilaxia ao ovo, reação prévia à VASPR e asma não controlada em doentes com alergia documentada ao ovo.

Objetivo: Caracterizar a população referenciada para administração da VASPR a nível hospitalar relativamente ao cumprimento das recomendações existentes, à segurança da administração da vacina em crianças com suspeita de alergia ao ovo e ao atraso na administração da primeira dose.

Tipo de estudo: Observacional transversal com colheita retrospetiva de dados.

Local: Serviço de Imunoalergologia do Hospital de Dona Estefânia.

População: Crianças referenciadas para administração hospitalar da VASPR.

Métodos: Consulta dos processos clínicos num período de cinco anos.

Resultados: Das 83 crianças referenciadas, $43 \%$ não apresentava qualquer sintoma com a ingestão de ovo. Entre os participantes que apresentavam sintomas dois tinham história de anafilaxia ao ovo e dois tinham asma brônquica controlada. Verificou-se um atraso global de dois meses no calendário vacinal. Nenhum dos doentes referenciados para a administração do reforço vacinal tinha história prévia de reação à VASPR. Não foram observadas reações sistémicas após a administração da vacina. Três doentes tiveram reação cutânea local e transitória. Conclusão: A maioria da população não apresentava alergia ao ovo, verificando-se um sobre diagnóstico de alergia alimentar. A administração da VASPR foi segura, mesmo nos casos com anafilaxia ao ovo. Após a publicação da orientação da DGS continuou a existir referenciação hospitalar injustificada, implicando atrasos significativos no calendário vacinal e sobrecarregando os serviços hospitalares.

Palavras-chave: Hipersensibilidade ao Ovo, Vacina Antisarampo, Parotidite e Rubéola.

\section{INTRODUÇÃO}

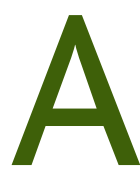
alergia ao ovo tem uma prevalência de 1,5 a 3,2\% na idade pediátrica, ${ }^{1}$ com aquisição de tolerância em $66 \%$ dos casos até aos cinco anos de idade. ${ }^{2} \mathrm{~A}$ VASPR (vacina contra o sarampo, parotidite epidémica e rubéola) inclui vírus cultivados em fibroblastos de embrião de galinha, podendo conter vestígios de ovalbumina, a proteína alergénica mais abundante da clara do ovo. Foi demonstrado, há 40 anos, que a quantidade de ovalbumina presente na vacina é de aproximadamente $0,01 \mu \mathrm{g},{ }^{3-4}$ um valor baixo e provavelmente subalergénico. ${ }^{5}$

Apesar dos vários estudos publicados a atestar a segurança da administração da VASPR em doentes com alergia ao ovo, ${ }^{4-11}$ continua a existir alguma hesitação na vacinação

'Médica Assistente Hospitalar, Serviço de Imunoalergologia do Hospital D. Estefânia, Centro Hospitalar Lisboa Central, EPE.

${ }^{2}$ Médica Assistente Hospitalar, Serviço de Imunoalergologia, Centro Hospitalar do Algarve, EPE.

${ }^{3}$ Médica Assistente Hospitalar Graduada Sénior, Serviço de Imunoalergologia do Hospital D. Estefânia, Centro Hospitalar Lisboa Central, EPE. de crianças com história clínica de reação ao ovo ou que, ainda que não o tenham introduzido na dieta, apresentem testes cutâneos (TC) ou doseamento de IgE específica positivos para o mesmo. Na dúvida, os doentes são referenciados para administração da VASPR em meio hospitalar, com consequentes atrasos no cumprimento do Programa Nacional de Vacinação (PNV). ${ }^{12}$

A Direção-Geral da Saúde (DGS) publicou uma orientação com as recomendações para a administração da VASPR, ${ }^{13}$ na qual refere que quer as crianças com alergia ao ovo, com reações que não sejam consideradas graves, quer as que ainda não introduziram o ovo na sua dieta devem ser vacinadas nos serviços de vacinação habitual.

As indicações para a vacinação em meio hospitalar incluem os casos em que há uma história pessoal documentada de anafilaxia ao ovo, uma reação prévia à administração da VASPR ou uma asma não controlada numa criança com história documentada de alergia ao ovo, independentemente da gravidade da reação prévia ao mesmo. 
Este estudo teve por objetivo caracterizar a população referenciada para administração da VASPR a nível hospitalar, relativamente ao cumprimento das recomendações existentes, à segurança da administração da vacina em crianças com suspeita de alergia ao ovo e ao atraso na administração da primeira dose.

\section{MATERIAL E MÉTODOS}

Efetuou-se uma colheita retrospetiva dos dados contidos nos processos clínicos de todos os doentes referenciados à consulta de imunoalergologia do Hospital de Dona Estefânia para administração da VASPR, durante um período de cinco anos (janeiro de 2008 a dezembro de 2012), com base no registo sistemático do agendamento do procedimento.

A população do estudo foi caracterizada relativamente às variáveis quantitativas contínuas, idade de vacinação, atraso na administração da primeira dose da vacina e às qualitativas nominais, sexo, sintomatologia que fez suspeitar da alergia ao ovo, resultados de TC com o ovo, doseamento de IgE para o ovo e reações adversas decorrentes da administração da vacina.

A amostra foi dividida em dois grupos. O primeiro incluiu as crianças referenciadas para a primeira administração da VASPR e o segundo as crianças referenciadas para a administração do reforço vacinal aos 5-6 anos.

O primeiro grupo foi subdividido de acordo com o PNV vigente à data da vacinação: no subgrupo 1A se a vacinação se deu antes de 2012 e no subgrupo 1B se a administração ocorreu após a alteração do PNV em 2012, quando a primeira dose da VASPR deixou de ser administrada aos 15 meses, passando a sê-lo aos 12 meses.

Por norma, a administração da vacina é feita em dose única e com um período de vigilância no serviço de 30 minutos a uma hora.

Foi feita análise descritiva dos dados com cálculo da média e desvio-padrão para as variáveis quantitativas e análise de frequências expressa em percentagem para as variáveis qualitativas, após caracterização.

\section{RESULTADOS}

Foram referenciadas 83 crianças (58 do sexo masculino). Destas, 53 incluíam-se no subgrupo 1A, 16 no subgrupo 1B e 14 no Grupo 2 (Quadro I).

A maioria da população $(58 / 83)$ não tinha história clínica de reação alérgica ao ovo. Nestes casos, o diagnóstico de alergia alimentar fez-se com base na positividade do doseamento de IgE para ovo em doentes com eczema atópico (35 doentes) e com sibilância recorrente (dois doen- tes), tendo-se verificado que os restantes 21 casos não apresentavam IgE ou TC positivos para ovo.

No grupo de doentes que referia sintomas após a ingestão de ovo (25/83), dois tinham antecedentes de anafilaxia, 19 queixas mucocutâneas e quatro sintomas gastrointestinais. Onze destes doentes ingeriam ovo regularmente quando foram vacinados com a VASPR.

À data da vacinação, 36 crianças toleravam a ingestão de ovo sem sintomas, 14 estavam em evicção alimentar de ovo por terem apresentado sintomas com a sua ingestão e em 33 doentes a tolerância ao ovo era desconhecida.

Nas situações em que a tolerância ao ovo era desconhecida foram incluídos os doentes que nunca tinham ingerido ovo (19/83) e os casos em que se desconhecia a idade de introdução do ovo na dieta (14/83). Nos motivos que levaram à evicção do ovo nos doentes que nunca o tinham ingerido encontravam-se IgE positiva para o ovo em doentes com eczema atópico (12/19), IgE positiva para o ovo em doentes com sibilância recorrente (2/19), diagnóstico de alergia a outros alimentos (2/19), IgE positiva para o ovo (2/19) e num caso não foi possível identificar o motivo.

Relativamente à presença de doenças alérgicas, dois doentes apresentavam asma brônquica controlada e 54 tinham eczema atópico, com doseamento de IgE para clara de ovo positivo em 37 casos.

Os TC com extrato comercial de ovo foram efetuados em 72 crianças, tendo sido positivos em 57.

O doseamento de IgE para o ovo foi efetuado em 59 doentes antes da referenciação hospitalar e foi positivo para a gema e para a clara em 36 e 54 casos, respetivamente.

A vacinação decorreu, em média, aos 18,3 meses no subgrupo 1A e aos 15,5 meses no subgrupo 1B, tendo-se verificado um atraso do calendário vacinal de dois e quatro meses, respetivamente. Apenas 12 em 53 doentes do subgrupo $1 \mathrm{~A}$ e três em 16 do subgrupo $1 \mathrm{~B}$ cumpriram os prazos estipulados no PNV.

No Grupo 2, como a vacina tem um prazo alargado de administração, podendo ser efetuada entre os cinco e os seis anos, apenas se verificou atraso num doente, no qual a vacina foi administrada aos sete anos. Neste grupo não havia referência a reação adversa à administração da primeira dose da VASPR.

Após a administração da vacina observou-se uma reação local transitória, com ligeiro eritema no local da administração, em 3/83 doentes, não tendo sido necessária qualquer medicação. Estas três crianças não referiam manifestações clínicas com ovo, encontrando-se em evicção por IgE positivas. Não se verificaram reações sistémicas, imediatas ou tar- 


\begin{tabular}{|c|c|c|c|}
\hline & Grupo 1A & Grupo 1B & Grupo 2 \\
\hline $\mathbf{N}$ & 53 & 16 & 14 \\
\hline $\begin{array}{l}\text { Idade - meses } \\
\text { Média } \pm \text { DP }\end{array}$ & $18,3 \pm 3,3$ & $15,5 \pm 2,8$ & $5,5 \pm 0,5$ anos \\
\hline $\begin{array}{l}\text { Atraso no PNV - meses } \\
\text { Mediana } \\
\text { P25-P75 }\end{array}$ & $\begin{array}{l}2,0 \\
1,0-4,8\end{array}$ & $\begin{array}{l}4,0 \\
1,0-6,0\end{array}$ & $\begin{array}{r}\text { Atraso de } 12 \text { meses } \\
\text { num doente }\end{array}$ \\
\hline $\begin{array}{l}\text { Tolerância ao ovo - } n(\%) \\
\text { Sim } \\
\text { Não } \\
\text { Desconhecida } \\
\text { - Sem introdução dieta } \\
\text { - Introdução desconhecida }\end{array}$ & $\begin{array}{l}22(41,0) \\
11(21,0) \\
20(38,0) \\
12(60,0) \\
8(40,0)\end{array}$ & $\begin{array}{l}6(37,5) \\
2(12,5) \\
8(50,0) \\
5(60,0) \\
3(40,0)\end{array}$ & $\begin{array}{r}8(57,0) \\
1(7,0) \\
5(36,0) \\
2(40,0) \\
3(60,0)\end{array}$ \\
\hline $\begin{array}{l}\text { Tipo sintomas }-n(\%) \\
\text { Mucocutâneos } \\
\text { Gastrointestinais } \\
\text { Anafilaxia }\end{array}$ & $\begin{array}{l}13(24,5) \\
3(6,0) \\
2(4,0)\end{array}$ & $\begin{array}{l}2(12,5) \\
1(6,0) \\
0\end{array}$ & $\begin{array}{r}4(29) \\
0 \\
0\end{array}$ \\
\hline $\begin{array}{l}\text { Doenças atópicas } \\
\text { Eczema } \\
\text { Asma } \\
\text { Rinite } \\
\end{array}$ & $\begin{array}{l}36 \\
0 \\
0\end{array}$ & $\begin{array}{l}11 \\
0 \\
0\end{array}$ & $\begin{array}{l}7 \\
2 \\
2\end{array}$ \\
\hline $\begin{array}{l}\text { TC ovo }-n(\%) \\
\text { Efetuados } \\
\text { Positivos } \\
\text { Negativos } \\
\end{array}$ & $\begin{array}{l}47(89,0) \\
38 \\
9\end{array}$ & $\begin{array}{l}12(75,0) \\
9 \\
3\end{array}$ & $\begin{array}{r}13(93,0) \\
10 \\
3\end{array}$ \\
\hline $\begin{array}{l}\text { IgE }-n(\%) \\
\text { Efetuada } \\
\text { Positiva gema } \\
\text { Positiva clara }\end{array}$ & $\begin{array}{l}41(77,0) \\
23 \\
31\end{array}$ & $\begin{array}{l}10(6,0 \%) \\
6 \\
16\end{array}$ & $\begin{array}{r}8(57,0) \\
7 \\
7\end{array}$ \\
\hline Reação local - $n$ (\%) & $3(6,0)$ & 0 & 0 \\
\hline
\end{tabular}

Legenda: TC: teste cutâneo por picada; IgE: Imunoglobulina E específica.

dias em nenhum dos doentes da amostra, incluindo os dois doentes que tinham tido história prévia de anafilaxia ao ovo.

\section{DISCUSSÃO}

Os dados deste trabalho estão de acordo com os resultados de outros estudos ${ }^{4-11}$ publicados na literatura acerca da segurança da administração daVASPR em doentes com suspeita ou com alergia confirmada ao ovo, nomeadamente os de uma casuística do mesmo serviço que foi apresentada num artigo de revisão. ${ }^{15}$

A diretiva relativa à referenciação hospitalar para administração da VASPR constante no PNV de $2006^{16}$ foi reforçada pela orientação da DGS de $2012,{ }^{12}$ em que a alergia ao ovo não contra-indica a administração da vacina, sendo uma falsa contra-indicação. No entanto, constata-se que a alergia ao ovo, ou a sua suspeição, tem continuado a motivar referenciação hospitalar para administração da VASPR.

Verificou-se atraso na primo vacinação em 54/69 doentes, o que vem confirmar que a referenciação hospitalar de todos os doentes que relatem uma história de alergia ao ovo ou que apresentem IgE ou TC positivos para o mesmo, poderá determinar atrasos no cumprimento do calendário vacinal, aumentando o risco de contrair as doenças cobertas pela vacina. ${ }^{17}$

Não está recomendado que se proceda, de forma sistematizada, a qualquer investigação alergológica antes da administração da vacina, nomeadamente realização de TC com ovo ou com a vacina ou o doseamento de IgE, em doentes que refiram queixas com a ingestão de ovo ou que ainda não o tenham introduzido na dieta. ${ }^{18}$ Nesta casuística, a maioria das crianças realizou TC com o extrato comercial de ovo por decisão médica individual, devido ao fácil acesso ao procedimento que permite uma avaliação rápida dos resultados.

Verificou-se um sobrediagnóstico de alergia alimentar, efetuado com base nos resultados de TC e de IgE, pois $43 \%$ das crianças não tinha alergia ao ovo, ingerindo-o sem queixas apesar dos exames positivos. A sensibilização a alimentos não é sinónimo de alergia alimentar. O valor preditivo positivo dos TC e da IgE no diagnóstico de alergia alimentaré baixo, com uma elevada ocorrência de falsos positivos. ${ }^{19}$ Os doentes com eczema atópico geram IgE para múltiplos alimentos, que podem não ter qualquer relevância clínica. ${ }^{20} \mathrm{~A}$ maioria da amostra (68\%) tinha eczema atópico, o que pode ter contribuído para este sobrediagnóstico de alergia alimentar.

Convém considerar que o tamanho da amostra é reduzido, o que limita a generalização dos resultados. Também não foi possível, por se tratar de uma análise retrospectiva de informação, determinar o tempo decorrido entre a referenciação hospitalar e a administração da vacina.

Em conclusão, a alergia ao ovo ou a sua suspeita continua a ser o principal motivo de referenciação hospitalar para vacinação com a VASPR, sendo que apenas duas das 83 crianças tinham história prévia de anafilaxia às proteínas do ovo. O encaminhamento de todos estes casos contribui para atrasos evitáveis na administração da vacina VASPR. 


\section{REFERÊNCIAS BIBLIOGRÁFICAS}

1. Lack G. Clinical practice: food allergy. N Engl J Med. 2008;359(12):1252-60.

2. Allen CW, Campbell DE, Kemp AS. Egg allergy: are all childhood food allergies the same? J Paediatr Child Health. 2007;43(4):214-8.

3. O'Brien TC, Maloney C), Tauraso NM. Quantitation of residual host protein in chicken embryo-derived vaccines by radial immunodiffusion. Appl Microbiol. 1971;21(4):780-2

4. Fasano MB, Wood RA, Cooke SK, Sampson HA. Egg hypersensitivity and adverse reactions to measles, mumps, and rubella vaccine. J Pediatr. 1992;120(6):878-81.

5. Hawkes CP, Mulcair S, Hourihane JO. Is hospital based MMR vaccination for children with egg allergy here to stay? Ir Med J. 2010;103(1):17-9.

6. Andersen DV, Jørgensen IM. MMR vaccination of children with egg allergy is safe. Dan Med J. 2013;60(2):A4573.

7. Torres Borrego J, Guzmán EG. Seguridad de la vacunación triple vírica en pacientes con alergía al huevo [Safety of MMR immunization in egg-allergic children]. An Pediatr (Barc). 2006;64(5):464-7. Spanish

8. Goodyear-Smith F, Wong F, Petousis-Harris H, Wilson E, Turner N. Follow-up of MMR vaccination status in children referred to a pediatric immunization clinic on account of egg allergy. Hum Vaccin. 2005;1(3):118-22.

9. Fina Avilés F, Campins Martí M, Martínez Gómez X, Rodrigo Pendás JA, Lushchenkova O, Pimós Tella L, et al. Vacuna triple vírica y alergia al huevo:experiencia en una unidad de vacunación hospitalária [[MMR vaccine and egg allergy: experience in a hospital immunization unit]. An Pediatr (Barc). 2007;67(4):362-7. Spanish

10. Ainsworth E, Debenham P, Carrol ED, Riordan FA. Referrals for MMR immunisation in hospital. Arch Dis Child. 2010;95(8):639-41.

11. Cronin J, Scorr A, Russell S, McCoy S, Walsh S, O'Sullivan R. A review of a paediatric emergency department vaccination programme for patients at risk of allergy/anaphylaxis. Acta Paediatr. 2012;101(9):941-5.

12. Direção-Geral da Saúde. Programa nacional de vacinação: norma n 40/2011 (atualização de 26/01/2012). Lisboa: DGS; 2012.
13. Direção-Geral da Saúde. Programa nacional de vacinação 2012, VASPR (vacina combinada contra o sarampo, a parotidite epidémica e a rubéola) e a alergia ao ovo: orientação n 006/2012 (atualização de 19/03/2012). Lisboa: DGS; 2012.

14. Direção-Geral da Saúde. Rede de referenciação hospitalar de imunoalergologia. Lisboa: DGS; 2003. ISBN 9726750903

15. Direção-Geral da Saúde. Programa nacional de vacinação 2006. Lisboa: DGS; 2005. ISBN 9726751365

16. Carvalho F, Agro J, Almeida MM, Rosado-Pinto JE. Imunizações e hipersensibilidade a proteínas de ovo. Acta Pediatr Port. 1998;29(6):563-9. Portuguese

17. Caubet JC, Rudzeviciene O, Gomes E, Terreehorst I, Brockow K, Eigenmann PA. Managing a child with possible allergy to vaccine. Pediatr Allergy Immunol. 2014;25(4):394-403.

18. Khakoo GA, Lack G. Guidelines for measles vaccination in egg-allergic children. Clin Exp Allergy. 2000;30(2):288-93.

19. Nowak-Wegrzyn A, Wesley Burks A, Sampson HA. Reactions to foods. In Adkinson Jr NF, Bochner BS, Burks AW, Busse WW, Holgate ST, Lemanske JR RF, et al, editors. Middleton's allergy: principles and practice. 8th ed. Saunders; 2013. p. 1310-1339. ISBN 9780323085939

20. Sampson HA. Food sensitivity and the pathogenesis of atopic dermatitis. J R Soc Med. 1997;90 Suppl 30:2-8.

\section{CONFLITO DE INTERESSES}

As autoras declaram não ter conflitos de interesses.

\section{ENDEREÇO PARA CORRESPONDÊNCIA}

Sónia Rosa

Hospital Dona Estefânia, Rua Jacinta Marto, 1169-045 Lisboa

E-mail: soniacrosa@gmail.com

Recebido em 10-03-2015

Aceite para publicação em 03-12-2015

\section{ABSTRACT}

\section{MEASLES, MUMPS AND RUBELLA VACCINATION IN CHILDREN WITH SUSPECTED EGG ALLERGY}

The General Directorate for Health (DGS) has published a recommendation for the administration of measles, mumps, rubella vaccine (MMR) in hospital for patients with a history of anaphylaxis due to egg allergy, previous reaction to MMR, and uncontrolled asthma in patients with documented egg allergy.

Objective: To characterize the population referred to hospital for MMR administration regarding compliance with existing referral recommendations, the safety of vaccination in children with suspected egg allergy, and the delay in the administration of the first dose. Type of study: Cross-sectional study of hospital records.

Local: Immuno-allergy Department of Dona Estefânia Hospital, Lisbon.

Population: Children referred to hospital for MMR administration.

Methods: Examination of clinical files over a 5-year period.

Results: Of the 83 children referred, $43 \%$ had no symptoms upon ingestion of egg. Among the patients who had symptoms, two had a history of anaphylaxis on exposure to eggs and two had controlled bronchial asthma. There was a mean delay of two months in the immunization schedule. None of the patients referred for an MMR booster vaccination had a previous history of reaction to MMR vaccination. No systemic reactions were observed after vaccine administration. Three patients had local, transient skin reactions.

Conclusions: The majority of patients referred for MMR vaccination in hospital did not have an allergy to eggs. We found over-diagnosis of food allergy. MMR vaccination was safe, even in cases of patients with a prior history of anaphylaxis on exposure to eggs. After the publication of the DGS recommendation, unjustified hospital referral continues. This causes significant delays in immunization and overloading of hospital services.

Keywords: Egg Hypersensitivity; Measles-mumps-rubella Vaccine. 